\title{
Cerebral Energy Metabolism and Oxygen State during Hypoxia in Neonate and Adult Dogs
}

\author{
S. NIOKA, B. CHANCE, D. S. SMITH, A. MAYEVSKY, M. P. REILLY, \\ C. ALTER, AND T, ASAKURA \\ Department of Biochemistry \& Biophysics [S.N., B.C., A.M., C.A.], Department of Anesthesiology [D.S.S.], \\ University of Pennsylvania. School of Medicine, and Division of Hematology, The Children's Hospital of \\ Philadelphia [M.P.R., T.A.]. Philadelphia, Pennsylvania 19104
}

\begin{abstract}
The relationship between a noninvasive determination of relative oxygen saturation of $\mathrm{Hb}$ circulating in brain tissue $\left(\mathrm{StO}_{2}\right)$ and energy metabolism was investigated with respect to age [dogs in three age groups ( 0 to 6d-old, 7- to 21-d-old, and adults)] and to severity of brain hypoxia using double beam spectroscopy of Hb deoxygenation and nuclear magnetic resonance spectroscopy of energy metabolism. The in vivo oxy-Hb dissociation was determined from the relationship between $\mathrm{StO}_{2}$ curve in the adult dog brain and sagittal sinus oxygen partial pressure during graded hypoxemia and found to be sigmoidal with an oxygen dissociation constant of $26.6 \mathrm{~mm} \mathrm{Hg}$. This agreed with an in vitro determination for oxygen dissociation constant of $28.2 \mathrm{~mm} \mathrm{Hg}$ in adult dog red cells. The arterial oxygen pressure at which brain $\mathrm{StO}_{2}$ was reduced by $50 \%$ was shifted toward the right with increasing age $(22.2,33.8$, and $40.8 \mathrm{~mm} \mathrm{Hg}$, respectively). This correlated with an in vitro oxygen dissociation constant of red cell Hb of 17.0, 22.3, and $28.2 \mathrm{~mm} \mathrm{Hg}$ in the three age groups, respectively. The phosphocreatine-inorganic phosphate ratio $\left(\mathrm{PCr} / \mathrm{P}_{i}\right)$ was used to relate changes in cellular energy metabolism daring hypoxia with changes in $\mathrm{StO}_{2}$. There was no change in $\mathrm{PCr} / \mathrm{P}_{i}$ when $\mathrm{StO}_{2}$ had decreased to $50 \%$ of the control value. However, when the brain $\mathrm{StO}_{3}$ had decreased to between 7 and $15 \%$, a reduction of $\mathrm{PCr} / \mathrm{P}_{\mathrm{i}}$ to $50 \%$ of the normoxic value occurred. The $\mathrm{StO}_{2}$ at which $\mathrm{PCr} / \mathrm{P}_{\mathrm{i}}$ falls should be considered a critical level of hypoxemia inasmuch as it represents a threshold of energy failure. There was no significant age dependence in the relationship between $\mathrm{StO}_{2}$ and $\mathrm{PCr} / \mathrm{P}_{\mathrm{i}}$. Neonates had much smaller intravascular oxygen gradients than those in adults at a critical level of hypoxemia. However, compared with adults, the 1- to 3-wk-old age group requires a similar $\mathrm{O}_{2}$ availability and extraction for brain metabolic survival. (Pediatr Res 28: 54-62, 1990)
\end{abstract}

\section{Abbreviations}

$\mathrm{StO}_{2}$, relative oxygen saturation of $\mathrm{Hb}$ circulating in tissue $\mathrm{SaO}_{2}$, arterial oxygen saturation of $\mathrm{Hb}$

$\mathrm{SvO}_{2}$, venous oxygen saturation of $\mathrm{Hb}$

$\mathrm{PaO}_{2}$, arterial oxygen pressure

$\mathrm{Pa}_{50}, \mathrm{PaO}_{2}$ at which $\mathrm{StO}_{2}$ is $50 \%$

$P_{\text {sfl, }}$ oxygen dissociation constant

$\mathrm{PCr}$, phosphocreatine

$P_{b}$, inorganic phosphate

$\mathbf{P C r} / \mathbf{P}_{\text {in }}$ phosphocreatine-inorganic phosphate ratio

Received December 11, 1989; accepted March 7, 1990.

Correspondence: Shoko Nioka, Department of Biochemisiry \& Biophysics,

University of Pennsylvania, School of Medicine, Philadelphia, PA 19104.

Supported in part by NIH grants HR 34004, NS 23859, and NS 22881, D.S. Smith was supported by HL 07286 .

\author{
pHi, intracellular pH \\ $\mathrm{PvO}_{2}$, sagittal sinus oxygen partial pressure \\ $\mathrm{PtvO}_{2}$, oxygen pressure in brain vasculature \\ ${ }^{31} \mathrm{P}$ NMR, phosphorus nuclear magnetic resonance \\ $\mathrm{FiO}_{2}$, fraction of inspired oxygen
}

Adult brain tissue appears to be considerably more sensitive to hypoxia or ischemia $(1,2)$ than neonatal brain tissue, which appears to tolerate relatively long periods of oxygen deprivation (3-6). For example, the duration of tolerance to anoxia in neonatal dogs is 10 times longer than in adult dogs (4). This agedependent tolerance to anoxia may be related to various factors including the oxygen affinities of fetal and adult $\mathrm{Hb}$, oxygen availability, oxygen consumption, oxygen gradients, and mitochondrial capacity. However, because of difficulties in measuring oxygen partial pressure in the tissue vasculature, no prior work has been done to elucidate the relationship between the oxygen state of the vasculature and the high neonatal tolerance to hypoxia. In studying cellular oxygen states, two primary considerations, oxygen delivery and oxidative phosphorylation, are key elements to be monitored. To determine oxygen delivery, we have studied the $\mathrm{StO}_{2}$ in the adult and neonate using double beam spectroscopy (7-9), inasmuch as this gives a global evaluation of oxygen delivery in relation to tissue oxygen demand. To measure the oxidative phosphorylation, we used ${ }^{31} \mathrm{P}$ NMR to determine $\mathrm{PCr} / \mathrm{P}_{\mathrm{i}}$, which is proportional to phosphorylation potential $(10)$ and is also a monitor of oxygen deprivation ( 1 , 11). The primary objective of our study was to investigate the relationship between the deoxygenation of high affinity fetal $\mathrm{Hb}$ and the diminution of $\mathrm{PCr}$ and phosphorylation potential that occurs during systemic hypoxemia.

The fundamental equation for maintenance of life as defined by Bernard (12) and Cannon (13) is the steady state, which can be expressed as the equality of energy conservation between generation of cell energy and its use for cell function. The greater the surfeit of supply compared with demand, the smaller the risk of a hypoxic catastrophe. Some features of neonatal metabolism resist hypoxia. Neonates exhibit a high mitochondrial cytochrome $\mathrm{aa}_{3}$ turnover for ATP generation, particularly at low oxygen tensions (14) and low ATP consumption (15). A critical factor that may enable the neonate brain to resist hypoxia is its ability to function at a low phosphorylation potential $\left(\mathrm{PCr} / \mathrm{P}_{\mathrm{i}}=\right.$ 1) (11). The concentration of mitochondrial enzymes for ATP generation increases with age (16) and, as a consequence, there is increased mitochondrial electron transport, ATP production, and capacity for oxygen consumption $(15,17,18)$. Thus maturation leads to an increase in phosphorylation potential, and the electrical activity increases as neuronal activity is needed. Oxygen gradients may also increase and this change may be a key in the differential sensitivity to neurologic damage between neonates and adults. 


\section{MATERIALS AND METHODS}

Animal Preparation and Experimental Protocol. The studies were approved by the institutional Animal Care and Use Committee. Eight adult dogs were anesthetized with $2 \%$ isoflurane ( $1 \%$ during the experiment), and 16 puppies $(0-$ to 6 - and $7-$ to 21-d-old) were anesthetized with fentanyl, $50 \mu \mathrm{g} / \mathrm{kg}$, droperidol, $2.5 \mathrm{mg} / \mathrm{kg}$ (both given intraperitoneally), and $\mathrm{N}_{2} \mathrm{O}$. Though different anesthetics were used in adults and puppies, both agents were used in relatively low doses. The animals were intubated or tracheotomized and mechanically ventilated. Body temperature was maintained with heating blankets and monitored using a nasopharyngeal probe. Mean arterial blood pressure was measured through a catheter in the femoral artery. A slow infusion of $5 \%$ dextrose in half normal saline was maintained during the experiment. In all subjects, arterial blood was obtained to measure $\mathrm{PaO}_{2}$ and $\mathrm{pH}$ at each level of hypoxemia. In adult dogs, sagittal sinus blood samples were obtained to measure $\mathrm{PvO}_{2}$ and $\mathrm{SvO}_{2}$. For these measurements, an 18- to 20-gauge cannula was inserted into the anterior sagittal sinus through a bur hole in front of the occipital process. To minimize extracranial blood contamination of the samples, blood flow from the posterior part of the sagittal sinus was blocked by a small piece of surgicel placed just posterior to the catheter (19). The blood from the anterior sagittal sinus flowed into a calibrated container and was returned to the animal with a pump. The ${ }^{31} \mathrm{P}$ NMR findings suggested that there was no interference with brain oxygenation due to this procedure.

Graded hypoxemia was induced from an $\mathrm{FiO}_{2}$ of 0.21 by decrements of 0.02 . PCr/ $\mathrm{P}_{\mathrm{i}}$ was determined at 4 -min intervals. When $\mathrm{PCr} / \mathrm{P}$, began to decrease, the $\mathrm{FiO}_{2}$ was decremented more slowly to avoid an abrupt further decrease of $\mathrm{PCr} / \mathrm{P}_{\mathrm{i}}$. If $\mathrm{PCr} / \mathrm{Pi}$ started to decrease abruptly, or mean arterial blood pressure declined to lower than 55 and $30 \mathrm{~mm} \mathrm{Hg}$ in adults and puppies, respectively, epinephrine was infused at a rate of 3 to $10 \mu \mathrm{g} / \mathrm{kg} /$ min to maintain a steady state of energy metabolism. Sodium bicarbonate ( $1 \mathrm{M}$ ) was administered to keep blood base deficit less than $5 \mathrm{mEq} / \mathrm{L}$.

Measurement Techniques. Double beam spectroscopy. A Johnson Foundation dual wavelength air turbine spectrometer (U. of Penn., Philadelphia, PA) using a Hamamatsu R928 photomultiplier (Hamamatsu, Japan) and a JY-type curved holographic grating monochromator at 850 and $782 \mathrm{~nm}$ (resolution $0.5 \mathrm{~nm}$ ) was used to determine oxy-and deoxy-Hb changes for studies in and in vitro. The light source was a $50 \mathrm{~W}$ halogen lamp. For studies in vivo, the light source and photomultiplier were coupled to the brain by $3-\mathrm{mm}$ diameter glass fiber bundles that were attached to the bare skull $1 \mathrm{~cm}$ apart at a $35-45^{\circ}$ angle with respect to each other. Preliminary studies demonstrated that this distance between the two light guides is necessary to provide increased light through the brain surface vasculature, which is more abundant in this area than inside the brain. The skull thickness in the adult was thinned to $2 \mathrm{~mm}$ to produce a skullto-brain distance similar to that of neonates. When the light guide was separated by $5 \mathrm{~cm}$, as in previous studies, a contribution by cytochrome oxidase to the signal was observed (20). By these operations, the incident light intensity distribution was focused on the vasculature in all age groups and the contribution of cytochrome oxidase signal at $850 \mathrm{~nm}$ was minimized (Nioka $\mathrm{S}$, unpublished observations).

The absorbance was calculated using the control (normoxia) light intensity as a reference. Because 782 and $850 \mathrm{~nm}$ are equibestic points, $\mathrm{Hb}$ oxygen saturation can be calculated by the following equation with the assumption that the path lengths of light at 850 and $782 \mathrm{~nm}$ are equal (21).

$$
\mathrm{OD}_{850-782}=\mathrm{L}(\Delta \epsilon)\left[\left(\mathrm{HbO}_{2}\right)-(\mathrm{dHb})\right]
$$

where $O D_{850-782}$ is the difference between $O D$ at $850 \mathrm{~nm}$ and that at $782 \mathrm{~nm}$; $\mathrm{L}$ is the light path length; $\Delta \epsilon$ is the difference of extinction coefficients between oxy- and deoxy-Hb at $850 \mathrm{~nm}$
( $\Delta \epsilon$ is also equal to the difference of the extinction coefficients of deoxy- and oxy-Hb at $782 \mathrm{~nm}$; $\mathrm{HbO}_{2}$ is oxy-Hb; and dHb is deoxy-Hb. This equation shows that $\mathrm{OD}_{850-782}$ measures $\left[\mathrm{HbO}_{2}\right]$ [which is the term for oxygen saturation of $\mathrm{Hb}\left(\mathrm{StO}_{2}\right)$ if total $\mathrm{Hb}$ concentration [THb] is constant].

The procedure for determining $\mathrm{StO}_{2}$ is as follows: At an $\mathrm{FiO}_{2}$ of 0.33 , the light intensities at 850 and $782 \mathrm{~nm}$ were equalized with a balancing circuit. Therefore, this condition was used to normalize the reference light intensity. The highest $\mathrm{OD}_{850-782}$ was obtained during the recovery period after hypoxia when $\mathrm{FiO}_{2}$ was 1.0 and was defined as $100 \% \mathrm{StO}_{2}$ (this value was considerably higher than that obtained with $\mathrm{FiO}_{2}$ of 1.0 without prior hypoxia). Previous experiments in this laboratory indicated that at this time period a very small deoxy-Hb peak was detectable at $760 \mathrm{~nm}$. All data points were normalized to the $100 \% \mathrm{StO}_{2}$ and the lowest $\mathrm{StO}_{2}$ at $\mathrm{FiO}_{2}=0$ (obtained at the end of the study). These two points of calibration produce a relative value for oxygen saturation of $\mathrm{Hb}$ (values of $\mathrm{StO}_{2}$ ) in the brain. This value of $\mathrm{StO}_{2}$ was correlated with $\mathrm{SvO}_{2}$ and $\mathrm{SaO}_{2}$, and thus shown to be closely related to oxygen saturation in the brain. The OD of the sum of the absorbance at 850 and $782 \mathrm{~nm}$ is a function of total $\mathrm{Hb}$ concentration and can be used to estimate changes in total $\mathrm{Hb}$ concentration.

We performed studies to measure $\mathrm{Hb}$ oxygen saturation in vitro using the same dual wavelength system to estimate the error of our in vivo $\mathrm{StO}_{2}$ measurements. For these studies, the light guides were attached to a container $(2.5 \mathrm{~cm}$ diameter by $3 \mathrm{~cm}$ depth), the inside of which was blackened. A $5 \%$ yeast suspension was used as a scattering media to model the scattering of brain tissue. This particular concentration of yeast had been previously determined to have light-scattering properties similar to the brain (22). Fully oxygenated dog red blood cells were added to the yeast solution and the changes in light intensity at 782 to 850 $\mathrm{nm}$ were measured as oxygen saturation decreased from 100 to $0 \%$. Control data from blood-free yeast solutions showed that there was no absorbance change as oxygen concentration was decreased, demonstrating that the optical changes were only related to changes in $\mathrm{Hb}$ saturation. Our experiments showed that $\mathrm{OD}_{850-782}$ increased by 11 and $37 \%$ when the hematocrit of red cell suspension was increased from 5 to 10 and $20 \%$, respectively. Assuming that the mean red cell volume in the brain tissue is $5 \%(23,24)$ and the maximum increase in blood volume is 2-fold, the maximal error for the calculation of $\mathrm{StO}_{2}$ would be $11 \%$.

NMR spectroscopy. A 2.1-Tesla NMR spectroscopy system (Otsuka-Electronics, Havertown, PA) was used to measure $\mathrm{PCr}$ and $P_{j}$. A two-turn copper surface coil $(2.5 \mathrm{~cm})$ was placed on the top of the skull. Shimming established a mean value of 0.2 ppm for the half-ht line width in the water peak. A near $90^{\circ}$ flip angle pulse was optimized between 40 and $60 \mu$ s for every dog. The radio frequency pulse at $35.8 \mathrm{MHz}$ was transmitted and received with the same coil and the pulse interval was $4 \mathrm{~s}$. After Fourier transformation, the spectra were phased and curve fitted to determine the areas of $\mathrm{PCr}$ and $\mathrm{P}_{i}$ (Fig. 1). $\mathrm{PCr} / \mathrm{P}_{i}$ was calculated from the ratio of the area of the $\mathrm{PCr}$ and $\mathrm{P}_{i}$ peaks and the ratio of the saturation factors of $\mathrm{PCr}$ and $\mathrm{P}_{i}$. The saturation factors for 4-s acquisition intervals were obtained from the spectra using $20-\mathrm{s}$ intervals, which provided unsaturated spectra.

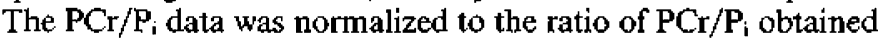
during the control period. $\mathrm{pHi}$ was calculated from the chemical shift of $\mathrm{P}_{\text {i }}$ referred to $\mathrm{PCr}$ (25). The ADP was calculated from creatine kinase equilibrium (26).

Measurement of blood gases and calculations of oxygen saturation of $\mathrm{Hb}$ in vitro. Blood gas tension and $\mathrm{pH}$ were measured with a Radiometer ABL2 (Copenhagen, Denmark). The blood $\mathrm{Hb}$ oxygen saturation curve was obtained with a Hemox AnaIyzer (TCS Medical Products Co., Huntington Valley, PA) (27) using blood taken from the femoral vein in all age groups. The oxygen saturation of $\mathrm{Hb}$ in the arterial and venous blood was 